\title{
HYPERBOLIC DYNAMICS IN NASH MAPS
}

\author{
R. A. BECKER, S. K. CHAKRABARTI, W. GELLER, B. KITCHENS, AND M. MISIUREWICZ
}

\begin{abstract}
We investigate the dynamics of the Nash better response map for a family of games with two players and two strategies. This family contains the games of Coordination, Stag Hunt and Chicken. Each map is a piecewise rational map of the unit square to itself. We describe completely the dynamics for all maps from the family. All trajectories converge to fixed points or period 2 orbits. We create tools that should be applicable to other systems with similar behavior.
\end{abstract}

\section{INTRODUCTION}

1.1. Economics introduction. Nash $[5,6,7]$ produced three proofs of the existence of an equilibrium point for $n$-person games. Each proof applied a fixed point theorem to a mapping from the Cartesian product of the players' mixed strategy sets to itself. Iteration of these maps lead to a discrete time dynamical systems on the product space of the players' mixed strategies.

Nash's [7] proof focused on a better response map with the property that each fixed point is an equilibrium point, and vice versa. Becker and Chakrabarti [2] generalized Nash's better response map to extend the existence theorem to incorporate some forms of nonexpected utility theories as well as to games with a continuum of pure strategies.

In [3] we analyzed the dynamics of the Nash map for Matching Pennies and showed that the game's equilibrium point was unstable - the players' mixed strategies converge to an orbit of period eight from any initial starting point except the mixed strategy equilibrium point. The Nash dynamics that one observes is quite different from what one gets if the players use their best responses. When the players use the best response map then the play simply cycles over the pure strategies.

The fact that the Nash map has interesting dynamics in the game of Matching Pennies raises questions about the dynamics of such behavior in other $2 \times 2$ games. Thus one would be curious about what would happen, say, in the case of the games of Coordination, Stag Hunt, or Chicken.

The purpose of this paper is to present a thorough analysis of the Nash better response dynamics for a one-parameter family of $2 \times 2$ games that include versions of the above games. We focus on the essential Nash map defined on the unit square. This map is derived from Nash's better response map by recognizing that it is sufficient to study the evolution of each players' probability of playing one of the available two pure strategies.

The payoff matrices that describe $2 \times 2$ games form an eight parameter family of matrices. Some of these matrices describe equivalent games under various notions of

Date: September 23, 2007.

M. Misiurewicz's research was partially supported by NSF grant DMS 0456526. 
equivalence. The payoff matrices we investigate here form a five parameter family and are described as follows.

\begin{tabular}{|c|c|c|}
\hline strategy & Left & Right \\
\hline Top & $\left(c+\alpha, b^{\prime}+\alpha\right)$ & $\left(b, b^{\prime}\right)$ \\
\hline Bottom & $\left(c, c^{\prime}\right)$ & $\left(b+\alpha, c^{\prime}+\alpha\right)$ \\
\hline
\end{tabular}

where $\alpha \geq 0$. All these games have the common feature that there are two pure strategy Nash equilibrium points, namely, (Top, Left) and (Bottom, Right) and a mixed strategy equilibrium. Some Coordination games belong to this class of games. Let $a=0=a^{\prime}, b=0=b^{\prime}$, and $c=0=c^{\prime}$. Then the payoff matrices become

\begin{tabular}{|c|c|c|}
\hline strategy & Left & Right \\
\hline Top & $(\alpha, \alpha)$ & $(0,0)$ \\
\hline Bottom & $(0,0)$ & $(\alpha, \alpha)$ \\
\hline
\end{tabular}

where $\alpha \geq 0$. Every game described by the five parameter family of matrices above has the same essential Nash map as a game described by a payoff matrix in this one parameter family (see Section 2, formulas (2.1) and (2.2)).

The following version of the Stag-Hunt game also fits our one-parameter family model (see [1] and [9]).

\begin{tabular}{|c|c|c|}
\hline strategy & Hunt Stag & Chase Rabbit \\
\hline Hunt Stag & $(4,4)$ & $(1,3)$ \\
\hline Chase Rabbit & $(3,1)$ & $(2,2)$ \\
\hline
\end{tabular}

The class of games also includes realizations of Chicken, as given below:

\begin{tabular}{|c|c|c|}
\hline strategy & Not Blink & Blink \\
\hline Not Blink & $(-10,-10)$ & $(5,-5)$ \\
\hline Blink & $(-5,5)$ & $(0,0)$ \\
\hline
\end{tabular}

If we interchange the strategy of one of the players then this game takes the required form with $\alpha=5$.

In our analysis of the Nash dynamics of this class of games we find that the nature of the dynamics related to the mixed strategy equilibrium point is sensitive to the payoffs of the game. For example, the mixed strategy equilibrium point can change from being an orientation preserving saddle point to an orientation reversing repelling point. This latter observation is especially interesting since, as far as we know, the existing literature on evolutionary games has not explicitly noted this phenomenon. This behavior cannot occur in the case of evolutionary dynamics in continuous time. The observations about the local instability of the pure strategy equilibrium is, however, quite consistent with results obtained in the literature on evolutionary dynamics in continuous time, as for example in [8].

1.2. Mathematics introduction. From the mathematical point of view, the problem we solve in the paper is to describe completely maps from a certain concrete one-parameter family $\mathcal{H}$ of maps of the unit square to itself. Problems of this type are often the most difficult ones in the whole theory of dynamical systems. While a lot of abstract theory exists, only a small part of this theory can be used in a concrete situation. Quite often the assumptions of general theorems are impossible to check. Sometimes the reasons why we cannot decide what is going on are deep. This 
applies especially to systems with complicated dynamics. How can one practically distinguish between a dense orbit and a periodic orbit of period $10^{100000000}$ ? However, for systems with relatively simple behavior a full description should be possible.

The systems considered in this paper fall into this category of "systems with relatively simple behavior," where after making some computations and drawing several computer pictures one can guess what is going on, but there is no obvious way to confirm it rigorously.

To resolve this problem, we create tools that allow us to prove exactly what we need. They are described in Section 4. They basically consist (after the standard preliminary use of symmetry to simplify the map) of several steps. The first one is to show that in the interesting region our map is an orientation preserving homeomorphism. The second step is to identify regions where the points are moved by the map in specific directions (cones of directions). This is basically treating our system as a difference equation. The third step is to see where the points from those regions can be mapped. Here we use the fact that under a homeomorphism the regions cannot "jump" over each other; the cyclic order around a point where several regions meet must stay the same.

We do not formalize our methods, although we believe that they can be used in many similar situations. However, each specific situation may require minor modifications, so it may be difficult to pinpoint exact assumptions that have to be made.

The computationally most difficult situations in the family $\mathcal{H}$ arise for parameter values for which bifurcations occur and the system is not hyperbolic. We do not consider them very important, and even omit them in the statement of Theorem 2.1, although we include them in Section 4 for completeness. Therefore the reader should not feel disturbed by formulas like those from the proof of Lemma 4.9 (starting with formulas (4.15) this proof ceases to be computational).

\section{Description of the FAmily $\mathcal{H}$ AND Results}

Let

$$
\left[\begin{array}{cc}
\left(a, a^{\prime}\right) & \left(b, b^{\prime}\right) \\
\left(c, c^{\prime}\right) & \left(d, d^{\prime}\right)
\end{array}\right]
$$

define a two person, two pure strategy game with $X$ the row player and $Y$ the column player. The two players have strategies $\bar{x}=(x, 1-x)$ and $\bar{y}=(y, 1-y)$, respectively. The payoff matrices for $X$ and $Y$ are

$$
R_{x}=\left[\begin{array}{ll}
a & b \\
c & d
\end{array}\right] \quad \text { and } \quad R_{y}=\left[\begin{array}{cc}
a^{\prime} & b^{\prime} \\
c^{\prime} & d^{\prime}
\end{array}\right] .
$$

The expected payoff for $X$ is $\bar{x} R_{x} \bar{y}^{T}$ and the expected payoff for $Y$ is $\bar{x} R_{y} \bar{y}^{T}$.

Define

$$
\begin{aligned}
t_{x} & =x+\max \left\{0,\left(e_{1}-\bar{x}\right) R_{x} \bar{y}^{T}\right\}, \\
t_{1-x} & =(1-x)+\max \left\{0,\left(e_{2}-\bar{x}\right) R_{x} \bar{y}^{T}\right\}, \\
t_{y} & =y+\max \left\{0, \bar{x} R_{y}\left(e_{1}-\bar{y}\right)^{T}\right\}, \\
t_{1-y} & =(1-y)+\max \left\{0, \bar{x} R_{y}\left(e_{2}-\bar{y}\right)^{T}\right\},
\end{aligned}
$$

with $e_{1}, e_{2}$ the standard basis vectors. 
The Nash map on the pair of probability vectors is

$$
(\bar{x}, \bar{y}) \mapsto\left(\left(\frac{t_{x}}{t_{x}+t_{1-x}}, \frac{t_{1-x}}{t_{x}+t_{1-x}}\right),\left(\frac{t_{y}}{t_{y}+t_{1-y}}, \frac{t_{1-y}}{t_{y}+t_{1-y}}\right)\right) .
$$

All information is contained in the essential Nash map on the unit square

$$
n=\left(n_{1}, n_{2}\right):[0,1]^{2} \rightarrow[0,1]^{2},
$$

defined by

$$
\begin{aligned}
& n_{1}(x, y)=\frac{t_{x}}{t_{x}+t_{1-x}}=\frac{x+\max \left\{0,\left(e_{1}-\bar{x}\right) R_{x} \bar{y}^{T}\right\}}{1+\max \left\{0,\left(e_{1}-\bar{x}\right) R_{x} \bar{y}^{T}\right\}+\max \left\{0,\left(e_{2}-\bar{x}\right) R_{x} \bar{y}^{T}\right\}}, \\
& n_{2}(x, y)=\frac{t_{y}}{t_{y}+t_{1-y}}=\frac{y+\max \left\{0, \bar{x} R_{y}\left(e_{1}-\bar{y}\right)^{T}\right\}}{1+\max \left\{0, \bar{x} R_{x}\left(e_{1}-\bar{y}\right)^{T}\right\}+\max \left\{0, \bar{x} R_{y}\left(e_{2}-\bar{y}\right)^{T}\right\}},
\end{aligned}
$$

with $\bar{x}$ and $\bar{y}$ as before. It is clear from the definition that in this setting the essential Nash map is a continuous map of the unit square into itself.

If we let $[r]^{+}=\max \{0, r\},[r]^{-}=\max \{0,-r\}$ and $\alpha=a-c, \beta=b-d, \gamma=a^{\prime}-b^{\prime}$, $\delta=c^{\prime}-d^{\prime}$, the essential Nash map reduces to

$$
\begin{aligned}
& n_{1}(x, y)=\frac{x+(1-x)[\alpha y+\beta(1-y)]^{+}}{1+(1-x)[\alpha y+\beta(1-y)]^{+}+x[\alpha y+\beta(1-y)]^{-}}, \\
& n_{2}(x, y)=\frac{y+(1-y)[\gamma x+\delta(1-x)]^{+}}{1+(1-y)[\gamma x+\delta(1-x)]^{+}+y[\gamma x+\delta(1-x)]^{-}} .
\end{aligned}
$$

Consequently, essential Nash maps arising from the 2 by 2 games form a four parameter family of piecewise rational, continuous maps of the unit square to itself. In what follows we examine the dynamics of a one parameter family $\mathcal{H}$ of essential Nash maps. It is given by $\alpha=\gamma>0$ and $\beta=\delta=-\alpha$.

The game of Coordination is defined by

$$
\left[\begin{array}{cc}
(1,1) & (-1,-1) \\
(-1,-1) & (1,1)
\end{array}\right]
$$

The essential Nash map for Coordination occurs in $\mathcal{H}$ with $\alpha=2$.

The game of Chicken is defined by

$$
\left[\begin{array}{cc}
(-10,-10) & (5,-5) \\
(-5,5) & (0,0)
\end{array}\right]
$$

Interchanging the ordering for $X$ 's choices one observes that the essential Nash map for Chicken occurs in $\mathcal{H}$ with $\alpha=5$.

An essential Nash map $n=\left(n_{1}, n_{2}\right) \in \mathcal{H}$ reduces to

$$
\begin{aligned}
& n_{1}(x, y)=\frac{x+\alpha(1-x)[2 y-1]^{+}}{1+\alpha(1-x)[2 y-1]^{+}+\alpha x[2 y-1]^{-}}, \\
& n_{2}(x, y)=\frac{y+\alpha(1-y)[2 x-1]^{+}}{1+\alpha(1-y)[2 x-1]^{+}+\alpha y[2 x-1]^{-}} .
\end{aligned}
$$

The diagonal is $\{(x, x): 0 \leq x \leq 1\}$. The region above the diagonal is $\{(x, y)$ : $0 \leq x<y \leq 1\}$ and the region below the diagonal is $\{(x, y): 0 \leq y<x \leq 1\}$. Reflection about the diagonal is given by the map $r_{d}(x, y)=(y, x)$. The anti-diagonal is $\{(x, 1-x): 0 \leq x \leq 1\}$. The region above the anti-diagonal is $\{(x, y): x+y>1\}$ 
and the region below the anti-diagonal is $\{(x, y): x+y<1\}$. Reflection about the anti-diagonal is given by $r_{a}(x, y)=(1-y, 1-x)$. A set $A$ is called invariant for a map $f$ if $f(A) \subseteq A$.

Let $f: U \rightarrow V$, where $U, V \subseteq \mathbb{R}^{2}$, be a local diffeomorphism. The map $f$ is orientation preserving at $p \in U$ if the Jacobian at $p$ is positive and orientation reversing at $p$ if the Jacobian is negative at $p$.

The main results about the one parameter family $\mathcal{H}$ of essential Nash maps are summarized in the following theorem. The reader can get slightly more information from Sections 3 and 4.

We will say that a point $x$ is attracted to $A$ (where $A$ can be a point or a compact set) if the trajectory of $x$ converges to $A$, that is, the distance from the $k$-th image of $x$ to $A$ converges to 0 as $k \rightarrow \infty$. When we say that a fixed point is orientation preserving or reversing, we mean the behavior of the map in a neighborhood of that point.

Theorem 2.1. Let $n \in \mathcal{H}$ be the essential Nash map described above.

(1) For all $\alpha,(0,0)$ and $(1,1)$ are orientation preserving, topologically attracting fixed points and $(1 / 2,1 / 2)$ is a fixed point. There are no other fixed points.

(2) When $0<\alpha \leq 1 / 2$, then $n$ is a homeomorphism onto its image. When $1 / 2<\alpha$, then $n$ is not one-to-one.

(3) For $0<\alpha<2,(1 / 2,1 / 2)$ is an orientation preserving fixed saddle point whose stable manifold is the anti-diagonal and unstable manifold is the diagonal (without $(0,0)$ and $(1,1))$.

(4) For $2<\alpha<4,(1 / 2,1 / 2)$ is an orientation reversing fixed saddle point whose stable manifold is the anti-diagonal and unstable manifold is the diagonal (without $(0,0)$ and $(1,1)$ ).

(5) For $\alpha \leq 4$, all points below the anti-diagonal are attracted to $(0,0)$ and all points above the anti-diagonal are attracted to $(1,1)$.

(6) For $4<\alpha<2(1+\sqrt{2}),(1 / 2,1 / 2)$ is an orientation reversing repelling fixed point. There is a saddle period two orbit on the anti-diagonal. One point of this orbit lies below $(1 / 2,1 / 2)$ and its stable manifold is the part of the antidiagonal with $x<1 / 2$. The other point lies above $(1 / 2,1 / 2)$ and its stable manifold is the part of the anti-diagonal with $x>1 / 2$. All points below the anti-diagonal are attracted to $(0,0)$ and all points above the anti-diagonal are attracted to $(1,1)$.

(7) For $\alpha>2(1+\sqrt{2}),(1 / 2,1 / 2)$ is an orientation reversing repelling fixed point. There is an attracting orbit of period two on the anti-diagonal. There are two saddle period two orbits that follow the orbit of period two on the antidiagonal. One saddle orbit lies below the anti-diagonal and one above. There are no other periodic points. Every other point is attracted to one of the periodic orbits mentioned (including $(0,0)$ and $(1,1)$ ). For illustration, see Figure 1.

The set of nonwandering points of each map in $\mathcal{H}$ consists of a finite number of periodic points. The points $(0,0)$ and $(1,1)$ are always topolgically attracting, but the derivative of the essential Nash map at these points is the identity. Except for the special values of $\alpha: 2,4$ and $2(1+\sqrt{2})$, and except for the points $(0,0)$ and $(1,1)$, 


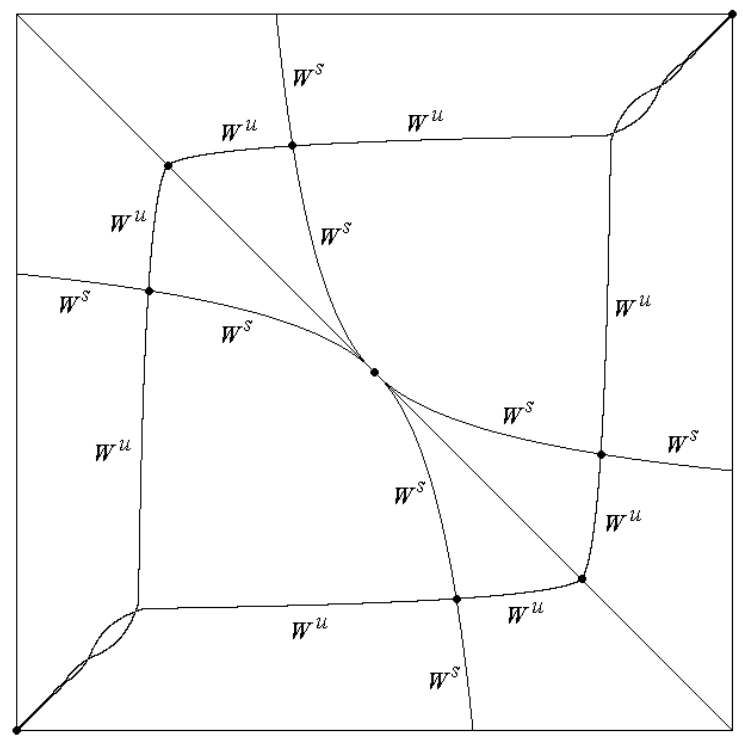

FiguRE 1. Periodic points for $\alpha=6$

all nonwandering points are hyperbolic periodic points. For this reason we refer to the dynamics as hyperbolic.

In Figure 1 we have marked all periodic points of $n$ for $\alpha=6$. Additionally we have marked the anti-diagonal and the stable and unstable manifolds of the period 2 saddles. Note that the stable manifolds serve as a boundary between the basins of attraction of the points $(0,0)$ and $(1,1)$ and the basin of attraction of the period 2 attracting orbit. The intersections between unstable manifolds are possible because the map $n$ is not a homeomorphism.

The four functions that occur in the essential Nash map simplify. We denote them as follows.

$$
\begin{array}{ll}
n_{1}^{+}(x, y)=\frac{x+\alpha(1-x)(2 y-1)}{1+\alpha(1-x)(2 y-1)} & \text { if } y \geq 1 / 2, \\
n_{1}^{-}(x, y)=\frac{x}{1+\alpha x(1-2 y)} & \text { if } y \leq 1 / 2, \\
n_{2}^{+}(x, y)=\frac{y+\alpha(1-y)(2 x-1)}{1+\alpha(1-y)(2 x-1)} & \text { if } x \geq 1 / 2, \\
n_{2}^{-}(x, y)=\frac{y}{1+\alpha y(1-2 x)} & \text { if } x \leq 1 / 2 .
\end{array}
$$

The two lines $x=1 / 2$ and $y=1 / 2$ divide the square into four quadrants. We refer to the quadrants by compass points. The northeast quadrant is $N E=\{(x, y)$ : $1 / 2 \leq x \leq 1,1 / 2 \leq y \leq 1\}$, and similar descriptions hold for the $S E, S W$ and $N W$ quadrants. In the $N E$ quadrant the essential Nash map is $n=\left(n_{1}^{+}, n_{2}^{+}\right)$, in the $S E$ quadrant it is $n=\left(n_{1}^{-}, n_{2}^{+}\right)$, in the $S W$ quadrant $n=\left(n_{1}^{-}, n_{2}^{-}\right)$and in the in the $N W$ quadrant $n=\left(n_{1}^{+}, n_{2}^{-}\right)$. We will refer to the lines separating the quadrants as borders. Also there are the North, South, East and West regions (see Figure 2). 


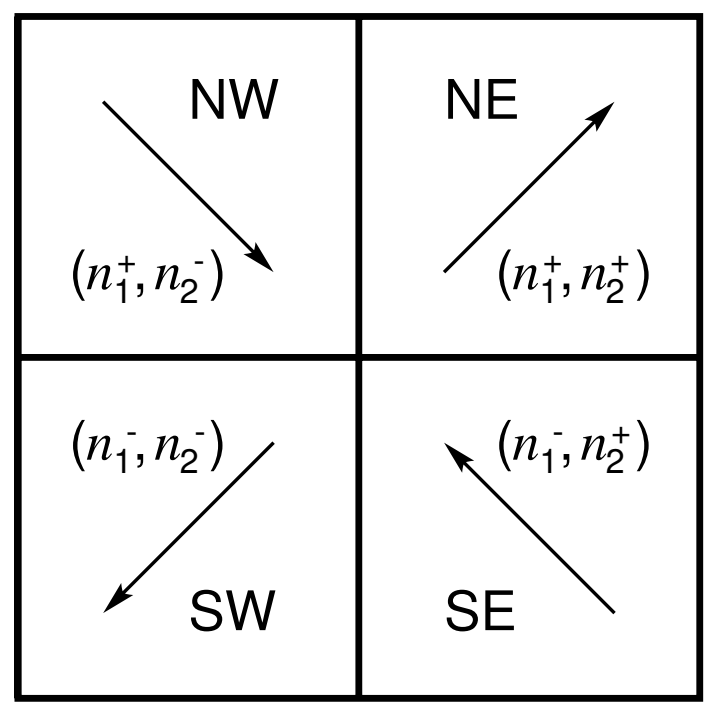

FiguRE 2. Quadrants, functions and directions

\section{Essential NASH MAP}

In this section we will investigate the essential Nash map $n$ in the whole square $[0,1]^{2}$.

Lemma 3.1. The essential Nash map $n$ commutes with $r_{d}$ and with $r_{a}$. Therefore both the diagonal and the anti-diagonal are invariant for $n$.

Proof. The first statement follows from a simple computation. The second part follows from the first one and the fact that the diagonal is the set of the fixed points of $r_{d}$ and the anti-diagonal is the set of the fixed points of $r_{a}$.

Lemma 3.2. Consider the essential Nash map acting on the unit square.

(1) The number $n_{1}(x, y)-x$ is positive if $x \neq 1$ and $y>1 / 2$, negative if $x \neq 0$ and $y<1 / 2$, and zero otherwise (including $y=1 / 2$ ). Similarly, the number $n_{2}(x, y)-y$ is positive if $y \neq 1$ and $x>1 / 2$, negative if $y \neq 0$ and $x<1 / 2$, and zero otherwise (including $x=1 / 2$ ). Loosely speaking, this means that under the action of the essential Nash map, all points in the open NE quadrant move to the northeast, all points in the open SE quadrant move to the northwest, all points in the open $S W$ quadrant move to the southwest, and all points in the open NW quadrant move to the southeast (see Figure 2).

(2) The essential Nash map has three fixed points. They are $(0,0),(1,1)$ and $(1 / 2,1 / 2)$.

(3) The northeast and southwest quadrants are invariant for $n$. Every point in the southeast quadrant, except $(1 / 2,1 / 2)$, is attracted to the point $(0,0)$ and every point in the northeast quadrant, except $(1 / 2,1 / 2)$, is attracted to the point $(1,1)$.

Proof. Statement (1) follows from simple computations. Statements (2) and (3) follow from (1). 
Lemma 3.3. The anti-diagonal and the regions above and below the anti-diagonal are invariant for $n$. Moreover, there exists a neighborhood $U$ of $(1 / 2,1 / 2)$ such that for every point $p \in U$ which does not lie on the anti-diagonal the distance of $n(p)$ from the anti-diagonal is larger than the distance of $p$ from the anti-diagonal.

Proof. We have

$$
n_{1}^{+}(x, y)+n_{2}^{-}(x, y)-1=(x+y-1) \frac{1+2 \alpha y(1-x)}{[1+\alpha(1-x)(2 y-1)][1+\alpha y(1-2 x)]}
$$

Since the fraction on the right is always positive, we see that if a point $p$ is above the anti-diagonal and in the $N W$ quadrant then $n(p)$ is above the anti-diagonal. Since $n$ commutes with $r_{d}$, we get the same result when we replace $N W$ by $S E$. The $N E$ quadrant is invariant by Lemma $3.2(3)$. Thus, the region above the anti-diagonal is invariant for $n$. Since $n$ commutes with $r_{a}$, the region below the anti-diagonal is also invariant for $n$. By continuity, the anti-diagonal is also invariant.

Using (3.1) and assuming that $x+y-1>0$, one can compute easily that

$$
n_{1}^{+}(x, y)+n_{2}^{-}(x, y)-1>x+y-1
$$

is equivalent to

$$
1-x-y+2 x y>\alpha(1-x)(1-2 x)(2 y-1) y .
$$

At $x=y=1 / 2$ the left-hand side of this inequality is equal to $1 / 2$, while the righthand side is 0 . Therefore the inequality holds in some neighborhood of $(1 / 2,1 / 2)$. Thus, if $p$ belongs to this neighborhood and lies above the anti-diagonal in the $N W$ quadrant, the distance of $n(p)$ from the anti-diagonal is larger than the distance of $p$ from the anti-diagonal. Using reflections about the anti-diagonal and diagonal and Lemma $3.2(1)$, we get a whole neighborhood $U$ of $(1 / 2,1 / 2)$ such that for every point $p \in U$ which does not lie on the anti-diagonal the distance of $n(p)$ from the anti-diagonal is larger than the distance of $p$ from the anti-diagonal.

We will need all eight partial derivatives of the four functions that define the essential Nash map for the discussion that follows. They are

$$
\begin{aligned}
\frac{\partial n_{1}^{+}}{\partial x} & =\frac{1}{[1+\alpha(1-x)(2 y-1)]^{2}}, \\
\frac{\partial n_{1}^{+}}{\partial y} & =\frac{2 \alpha(1-x)^{2}}{[1+\alpha(1-x)(2 y-1)]^{2}}, \\
\frac{\partial n_{1}^{-}}{\partial x} & =\frac{1}{\left[(1+\alpha x(1-2 y)]^{2}\right.}, \\
\frac{\partial n_{1}^{-}}{\partial y} & =\frac{2 \alpha x^{2}}{\left[(1+\alpha x(1-2 y)]^{2}\right.}, \\
\frac{\partial n_{2}^{+}}{\partial x} & =\frac{2 \alpha(1-y)^{2}}{[1+\alpha(1-y)(2 x-1)]^{2}}, \\
\frac{\partial n_{2}^{+}}{\partial y} & =\frac{1}{[1+\alpha(1-y)(2 x-1)]^{2}},
\end{aligned}
$$




$$
\begin{aligned}
\frac{\partial n_{2}^{-}}{\partial x} & =\frac{2 \alpha y^{2}}{[1+\alpha y(1-2 x)]^{2}} \\
\frac{\partial n_{2}^{-}}{\partial y} & =\frac{1}{[1+\alpha y(1-2 x)]^{2}}
\end{aligned}
$$

We know from Lemma $3.2(3)$ that the fixed points $(0,0)$ and $(1,1)$ are topological attractors (although from the differentiable point of view they are neutral; it is easy to check that the derivative at them is the identity). Let us investigate the nature of the third fixed point, $(1 / 2,1 / 2)$.

Lemma 3.4. (1) All four derivatives of the essential Nash map at $(1 / 2,1 / 2)$ are

$$
\left[\begin{array}{ll}
1 & \frac{\alpha}{2} \\
\frac{\alpha}{2} & 1
\end{array}\right]
$$

(2) The above matrix has two eigenvalues. The larger in modulus is $1+\frac{\alpha}{2}$ with eigenvector $(1,1)^{T}$ and the smaller in modulus is $1-\frac{\alpha}{2}$ with eigenvector $(1,-1)^{T}$.

(3) When $0<\alpha<2,(1 / 2,1 / 2)$ is an orientation preserving saddle point. When $2<\alpha<4,(1 / 2,1 / 2)$ is an orientation reversing saddle point. When $4<\alpha$, $(1 / 2,1 / 2)$ is an orientation reversing repelling fixed point.

Proof. Statements (1) and (2) are computations. Statement (3) follows immediately from the first two statements.

Let us turn to the investigation of the behavior of $n$ on the diagonal and antidiagonal.

Lemma 3.5. (1) The diagonal is mapped by $n$ homeomorphically onto itself.

(2) The anti-diagonal is mapped by $n$ homeomorphically into itself if and only if $\alpha \leq 1 / 2$ (then orientation is preserved) or if $\alpha \geq 2$ (then orientation is reversed).

(3) The upper and lower halves of the diagonal are invariant for $n$.

(4) The upper and lower halves of the anti-diagonal are invariant for $n$ if and only if $\alpha \leq 1$.

Proof. The essential Nash map restricted to the diagonal in the $N E$ quadrant is given by

$$
x \mapsto \frac{x+\alpha(1-x)(2 x-1)}{1+\alpha(1-x)(2 x-1)}
$$

Its derivative is

$$
\frac{1+2 \alpha(1-x)^{2}}{[1+\alpha(1-x)(2 x-1)]^{2}}>0
$$

and both endpoints of this segment are fixed points of $n$. In the $S W$ quadrant the situation is similar because of the symmetry with respect to the anti-diagonal. This proves statements (1) and (3).

The essential Nash map restricted to the anti-diagonal in the $N W$ quadrant is given by

$$
y \mapsto \frac{y}{1+\alpha y(2 y-1)}
$$


Its derivative is

$$
\frac{1-2 \alpha y^{2}}{[1+\alpha y(2 y-1)]^{2}} \text {. }
$$

If $\alpha \leq 1 / 2$ then the derivative above is positive for all $y \in[1 / 2,1)$, if $\alpha \geq 2$ then it is negative for all $y \in(1 / 2,1]$, and if $1 / 2<\alpha<2$ then it changes sign in $(1 / 2,1)$. In the $S E$ quadrant the situation is similar because of the symmetry with respect to the diagonal. This proves statement (2).

To prove statement (4), note that for $y>1 / 2$

$$
\frac{y}{1+\alpha y(2 y-1)} \geq \frac{1}{2}
$$

is equivalent to $\alpha y \leq 1$, which holds for all $y \in[1 / 2,1]$ if and only if $\alpha \leq 1$ (and the situation is similar in the $S E$ quadrant).

Let us return to the global view on the essential Nash map. We will use the following lemma (see [4], cf. [3]).

Lemma 3.6. Suppose that $C$ is a Jordan curve in the plane and $D$ is the closure of the region bounded by $C$. Let $f$ be a map from $D$ into $\mathbb{R}^{2}$, which is a local homeomorphism on the interior $D^{\circ}$ of $D$ and a homeomorphism from $C$ onto its image. Then:

(1) $f(C)$ is a Jordan curve and $f\left(D^{\circ}\right)$ is the region bounded by $f(C)$;

(2) $f$ is a homeomorphism of $D$ onto its image.

Lemma 3.7. If $\alpha \leq 1 / 2$ then $n$ is a homeomorphism onto its image.

Proof. Assume that $\alpha \leq 1 / 2$. By Lemma 3.1 it follows that it is enough to prove that $n$ restricted to the triangle $T$ with the vertices $(0,1),(1,1)$ and $(1 / 2,1 / 2)$ is a homeomorphism onto its image and that $n(T) \subseteq T$. From the formulas for the partial derivatives of $n$ we see that the Jacobian of $n$ in the $N W$ quadrant is a fraction with a positive denominator and the numerator equal to $1-4 \alpha^{2}(1-x)^{2} y^{2}$. Thus, this Jacobian is positive except at $(0,1)$, where it is zero. Similarly, the Jacobian of $n$ in the $N E$ quadrant is a fraction with a positive denominator and the numerator equal to $1-4 \alpha^{2}(1-x)^{2}(1-y)^{2}$, so it is positive everywhere. Therefore by Lemma 3.6 it remains to check that the image of the boundary of $T$ is contained in $T$ and that $n$ restricted to this boundary is a homeomorphism.

Let us figure out how $n$ acts on the three segments that comprise the boundary of $T$. By Lemma 3.5, $n$ restricted to the segment joining $(0,1)$ with $(1 / 2,1 / 2)$ is a homeomorphism onto its subsegment, and $n$ maps the segment joining $(1 / 2,1 / 2)$ with $(1,1)$ homeomorphically onto itself. In the $N W$ quadrant we have

$$
n(x, 1)=\left(\frac{x+\alpha(1-x)}{1+\alpha(1-x)}, \frac{1}{1+\alpha(1-2 x)}\right) .
$$

The second coordinate of the image above is a 1-to- 1 function of $x$, so $n$ restricted to the segment $I$ joining $(0,1)$ with $(1 / 2,1)$ is a homeomorphism. By Lemma 3.3 the image lies above the anti-diagonal (except the point $n(0,1)$ ).

To check that it lies above the diagonal, note that it is equivalent to

$$
\frac{x+\alpha(1-x)}{1+\alpha(1-x)}<\frac{1}{1+\alpha(1-2 x)},
$$


which is in turn equivalent to

$$
\alpha^{2}(1-2 x)(1-x)+\alpha x(1-2 x)+(x-1)<0 .
$$

If $x=1 / 2$, this is true. Fix $x \in[0,1 / 2)$ and look at the expression above as a quadratic polynomial of $\alpha$. The coefficients by $\alpha^{2}$ and $\alpha$ are positive, so the maximal value (for $\alpha \in(0,1 / 2]$ ) is attained at $\alpha=1 / 2$. Then the value of this polynomial is $\left(-2 x^{2}+3 x-3\right) / 4<0$, so $n(I)$ lies above the diagonal.

On the segment $J$ joining $(1 / 2,1)$ with $(1,1)$ we have $n_{2}^{+}(x, 1)=1$, so the image is contained in $I \cup J$. In particular, one eigenvector of the derivative of $n$ is horizontal. The Jacobian there is positive, so the corresponding eigenvalue is non-zero. Therefore $n$ restricted to $J$ is a homeomorphism onto its image. The set $n(J)$ intersects the diagonal only at $(1,1)$. Moreover, the image of $I$ intersects the line $y=1$ only at $n(1 / 2,1)$. This completes the proof.

Lemma 3.2 (3) tells us what happens to the trajectories of points that fall into the $N E$ or $S W$ quadrant. Let us consider other possibilities.

Lemma 3.8. If the whole trajectory of a point $p$ stays in the $N W$ quadrant then $p$ belongs to the anti-diagonal and is attracted to $(1 / 2,1 / 2)$. Similarly, if the whole trajectory of a point $p$ stays in the SE quadrant then $p$ belongs to the anti-diagonal and is attracted to $(1 / 2,1 / 2)$.

Proof. Assume that the whole trajectory of a point $p$ stays in the $N W$ quadrant. By Lemma 3.2 (1), along the trajectory both coordinates change in a monotone way. Therefore the trajectory converges to a fixed point. The only fixed point in the $N W$ quadrant is $(1 / 2,1 / 2)$, so the trajectory converges to this point. By Lemma 3.3, when the points on the trajectory are sufficiently close to $(1 / 2,1 / 2)$, they have to lie on the anti-diagonal. Since by Lemma 3.3 both the regions below and above the anti-diagonal are invariant, already $p$ had to lie on the anti-diagonal.

The similar statement for the $S W$ quadrant follows from the symmetry of the map with respect to the diagonal.

\section{Making USE OF The SyMmetry}

By the results of the preceding section, the only trajectories with unknown behavior are those that go through both $N W$ and $S E$ quadrants. In order to investigate them, we consider $r_{d} \circ n$ in the $N W$ and $S E$ quadrants. This map commutes with $r_{d}$ because $n$ does. Therefore, again looking at the $N W$ and $S E$ quadrants will be equivalent. In order to simplify further computations, we conjugate this map via the affine map $s(x, y)=(1-2 x, 2 y-1)$. It maps the $N W$ quadrant to the whole square $[0,1]^{2}$, the fixed point $(1 / 2,1 / 2)$ is mapped to $(0,0)$, and the anti-diagonal is mapped to the diagonal. The formula for our new map $f=s \circ r_{d} \circ n \circ s^{-1}$ in $[0,1]^{2}$, that we have to investigate, is

$$
f(x, y)=\left(\frac{-2 y+\alpha x(1+y)}{2+\alpha x(1+y)}, \frac{-2 x+\alpha y(1+x)}{2+\alpha y(1+x)}\right) .
$$

We will use notation $f(x, y)=\left(x^{\prime}, y^{\prime}\right)$.

Lemma 4.1. If $\alpha \leq 2$ then for every $(x, y) \in[0,1]^{2}$, except the fixed point $(0,0)$, we have $x^{\prime}<x$ and $y^{\prime}<y$. 
Proof. The inequality $x^{\prime}<x$ can be written as

$$
\frac{-2 y+\alpha x(1+y)}{2+\alpha x(1+y)}<x
$$

and this is equivalent to

$$
(1+y)\left(\alpha x^{2}-\alpha x+2\right)>2(1-x) .
$$

Since $\alpha \leq 2$, we have for $x>0$

$$
\alpha x^{2}>0 \geq(\alpha-2) x
$$

and if $x=0$, there is an equality. Therefore

$$
\alpha x^{2}-\alpha x+2>2(1-x)
$$

with equality for $x=0$, and (4.1) follows for $(x, y) \neq(0,0)$.

The same computations with $x, y$ switched give us $y^{\prime}<y$.

Let us return to the map $n$. The next lemma reduces the set of trajectories with unknown behavior to the ones that alternate (regularly) between the $N W$ and $S E$ quadrants.

Lemma 4.2. Assume that $\alpha>2$. If $(x, y) \neq(1 / 2,1 / 2)$ belongs to the $N W$ quadrant then $n(x, y)$ does not belong to the $N W$ quadrant. Similarly, if $(x, y) \neq(1 / 2,1 / 2)$ belongs to the SE quadrant then $n(x, y)$ does not belong to the SE quadrant.

Proof. Suppose that the point $(x, y) \neq(1 / 2,1 / 2)$ and its image both belong to the $N W$ quadrant. Then

$$
0 \leq x \leq 1 / 2 \leq y \leq 1
$$

and

$$
\frac{x+\alpha(1-x)(2 y-1)}{1+\alpha(1-x)(2 y-1)} \leq 1 / 2 \leq \frac{y}{1-\alpha y(2 x-1)} .
$$

The first inequality of $(4.3)$ is equivalent to $\alpha(1-x)(2 y-1) \leq 1-2 x$, and the second one to $\alpha y(1-2 x) \leq 2 y-1$. From this and (4.2) we get

$$
\alpha(1-x) \leq \frac{1-2 x}{2 y-1} \leq \frac{1}{\alpha y} .
$$

Thus, $(1-x) y \leq 1 / \alpha^{2}$. Together with $(4.2), \alpha>2$ and $(x, y) \neq(1 / 2,1 / 2)$, this gives us a contradiction.

Lemma 4.3. If $2<\alpha \leq 3+2 \sqrt{2}$ then the region $R$ of the square $[0,1]^{2}$ where $x^{\prime}>x$ is bounded from below by the segment of the lower side of the square from $x=0$ to $x=(\alpha-2) / \alpha$ and from above by the graph of

$$
y=\frac{2(1-x)}{\alpha x^{2}-\alpha x+2}-1 .
$$

If $\alpha>3+2 \sqrt{2}$ then $R$ is bounded from below by the segment of the lower side of the square from $x=0$ to $x=(\alpha-2) / \alpha$, from the left and right by the pieces of the graph of (4.4), and from above by the segment of the upper side of the square from

$$
x=\frac{\alpha-1-\sqrt{1-6 \alpha+\alpha^{2}}}{2 \alpha}
$$


to

$$
x=\frac{\alpha-1+\sqrt{1-6 \alpha+\alpha^{2}}}{2 \alpha} .
$$

At the points of the boundary of $R$ where (4.4) holds we have $x^{\prime}=x$. In the rest of $[0,1]^{2}$ we have $x^{\prime}<x$.

The same statements are true if we switch $x$ with $y$ and $x^{\prime}$ with $y^{\prime}$.

Proof. As we already noticed in the proof of Lemma 4.1, the inequality $x^{\prime}>x$ is equivalent to

$$
(1+y)\left(\alpha x^{2}-\alpha x+2\right)<2(1-x) .
$$

Moreover, $x^{\prime}=x$ is equivalent to (4.4). If $\alpha x^{2}-\alpha x+2>0$, then (4.5) is equivalent to

$$
y<\frac{2(1-x)}{\alpha x^{2}-\alpha x+2}-1
$$

Let us look closer at (4.4). We have $y=0$ when $x=0$ or $x=(\alpha-2) / \alpha$. If $\alpha<8$ then $\alpha x^{2}-\alpha x+2>0$, so $y>0$ is equivalent to $0<x<(\alpha-2) / \alpha$. If $\alpha \geq 8$ then the solutions to $\alpha x^{2}-\alpha x+2=0$ are

$$
x=\frac{1}{2}\left(1 \pm \sqrt{1-\frac{8}{\alpha}}\right) .
$$

We have

$$
0<\frac{1}{2}\left(1-\sqrt{1-\frac{8}{\alpha}}\right)<\frac{1}{2}\left(1+\sqrt{1-\frac{8}{\alpha}}\right)<\frac{\alpha-2}{\alpha} .
$$

The last inequality above follows from the inequality $(\alpha-2) / \alpha>1 / 2$ and the fact that the value of the polynomial $\alpha x^{2}-\alpha x+2$ at $x=(\alpha-2) / \alpha$ is $4 / \alpha>0$.

Next we differentiate the function given by (4.4). We get

$$
\frac{d y}{d x}=\frac{2\left(\alpha x^{2}-2 \alpha x+\alpha-2\right)}{\left(\alpha x^{2}-\alpha x+2\right)^{2}} .
$$

Thus, if $0 \leq x<1-\sqrt{2 / \alpha}$ then $d y / d x>0$ and if $1-\sqrt{2 / \alpha}<x \leq 1$ then $d y / d x<0$ (except at the points where the denominator is 0 ). Note that if $\alpha \geq 8$ then

$$
\frac{1}{2}\left(1-\sqrt{1-\frac{8}{\alpha}}\right) \leq 1-\sqrt{\frac{2}{\alpha}} \leq \frac{1}{2}\left(1+\sqrt{1-\frac{8}{\alpha}}\right) .
$$

Putting all this together we get the following description of the region $R$. It is bounded from below by the segment of the lower side of the square from $x=0$ to $x=(\alpha-2) / \alpha$. If the graph of (4.4) on this interval fits in the square, it bounds $R$ from above. This happens when (4.4) with $y=1$ has no solutions or one solution. A simple computation shows that this is when $\alpha \leq 3+2 \sqrt{2}$. For larger values of $\alpha$, up to 8, the graph of (4.4) goes out of the square through the upper side, and we get a description of $R$ as in the statement of the lemma (simple computations give the values of $x$ for which this graph intersects the upper side of the square). If $\alpha \geq 8$ then this description is still valid, since as the denominator of the right-hand side of (4.4) goes to 0, $y$ goes to infinity; the part of the graph of (4.4) between the values of $x$ where the denominator is infinity does not count because there $\alpha x^{2}-\alpha x+2<0$. 
The last statement of the lemma follows from the symmetry of $f$ with respect to the diagonal.

Lemma 4.4. If $\alpha \leq 4$ then for the map $n$ all points below the anti-diagonal are attracted to $(0,0)$ and all points above the anti-diagonal are attracted to $(1,1)$.

Proof. By Lemma 3.2 (3) and Lemma 3.1, it is sufficient to prove that the trajectories of all points of the $N W$ quadrant above the anti-diagonal converge to $(1,1)$.

If $\alpha \leq 2$ then by Lemmas 3.2 (1) and 4.1 as we move along such a trajectory then the distance from the diagonal decreases. Thus, either the trajectory enters the $N E$ quadrant (and then it converges to $(1,1))$ or it converges to $(1 / 2,1 / 2)$. However, the latter is impossible by Lemma 3.3.

If $2<\alpha \leq 4$ then by Lemma 4.2 in order to find out how the trajectory of a point $p$ behaves it is enough to analyze the trajectory of $s(p)$ under $f$. To do this we will show that the region $R$, described in Lemma 4.3, lies below the diagonal. Then its reflection from the diagonal lies above the diagonal, and since $s(p)$ also lies above the diagonal, on the trajectory of $s(p)$ for $f$ the first coordinate decreases. Thus, this trajectory either gets out of the square or it converges to $(0,0)$. Therefore the trajectory of $p$ for $n$ either enters the $N E$ quadrant (and then it converges to $(1,1)$ ) or it converges to $(1 / 2,1 / 2)$. Again, the latter is impossible by Lemma 3.3.

To show that $R$ lies below the diagonal, we note that if this is not the case then the graph of (4.4) intersects the diagonal at some point other than $(0,0)$. However, if the right-hand side of (4.4) is equal to $x$ and $x \neq 0$ then $\alpha x^{2}=\alpha-4$, and this has no solution (with $x \neq 0$ ) if $\alpha \leq 4$. This completes the proof.

Let us illustrate the dynamics of $f$. We mark the region $R$ and the region $\widetilde{R}$ symmetric to it with respect to the diagonal, the regions which are mapped outside the square, the fixed points inside the square, and approximate directions in which the points move (that is, approximate directions of vectors $f(p)-p)$. The formulas for the regions which are mapped outside the square are simple. Since the denominators in the formula for $f$ are positive, $x^{\prime}<0$ is equivalent to

$$
x<\frac{2 y}{\alpha(1+y)} .
$$

To get the inequality equivalent to $y^{\prime}<0$ we have to switch $x$ and $y$. Figure 3 illustrates the case $2<\alpha \leq 4$.

Let us now investigate the dynamics of $n$ for $\alpha>4$. As we already noticed, Lemma 3.2 (3) tells us everything about the behavior of the trajectories that enter the $N E$ or $S W$ quadrants, and by Lemma 4.2 in the remaining two quadrants in order to understand the dynamics of $n$ it is enough to understand the dynamics of $f$ in $[0,1]^{2}$. In particular, except the point $(0,0)$, all fixed points of $f$ correspond to period 2 orbits of $n$. Therefore, let us start with investigating the fixed points of $f$.

We will need the following simple lemma.

Lemma 4.5. Let $T$ be the trace and $D$ the determinant of a 2 by 2 matrix $A$. Then $A$ has two real eigenvalues, one larger than 1 and another smaller than 1 , if and only if $T>D+1$.

Proof. The equation for the eigenvalues of $A$ is $x^{2}-T x+D=0$. Its discriminant is $\Delta=T^{2}-4 D$, and the solutions are $(T \pm \sqrt{\Delta}) / 2$. They are both real, one larger 

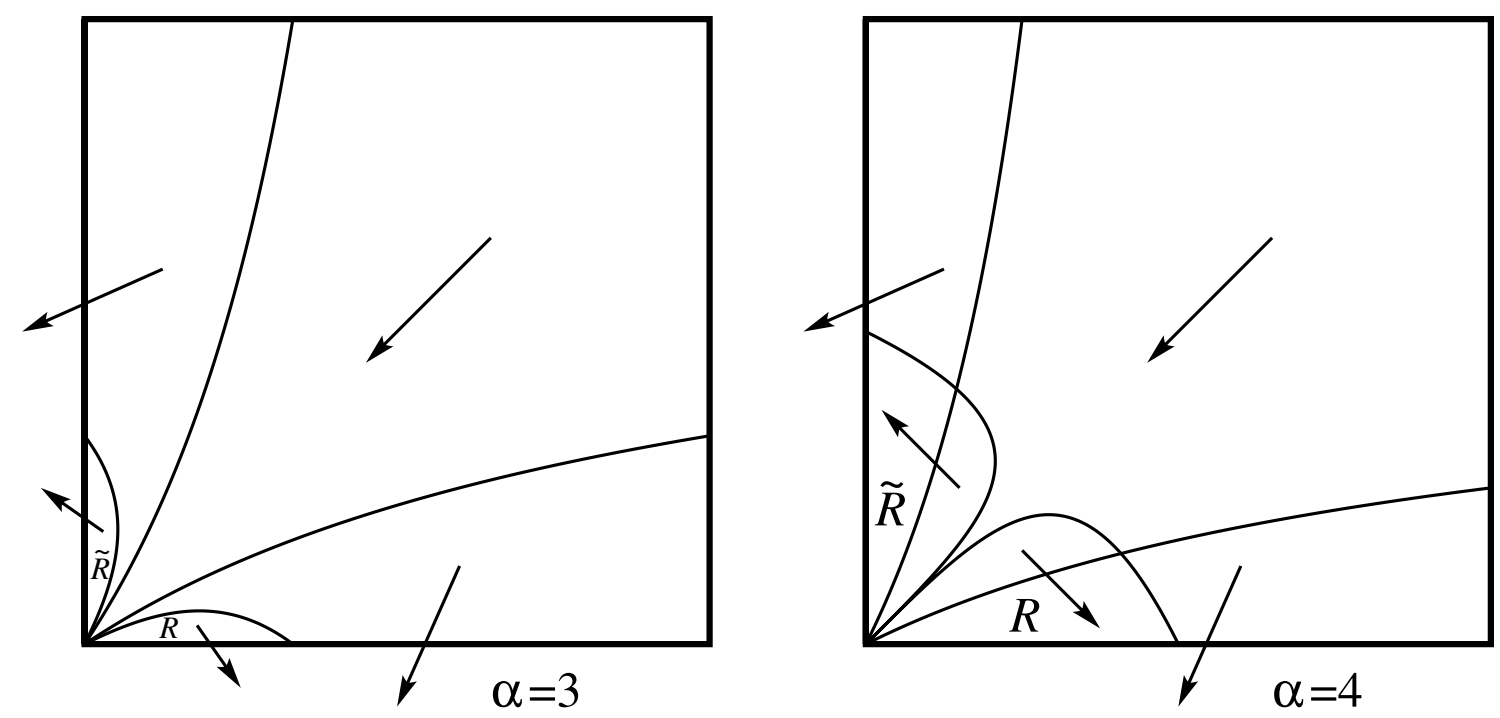

Figure 3. Map $f$ for $\alpha=3$ and $\alpha=4$

than 1 and another smaller than 1 , if and only if $\Delta>0$ and

$$
\frac{T-\sqrt{\Delta}}{2}<1<\frac{T+\sqrt{\Delta}}{2}
$$

which is equivalent to $\sqrt{\Delta}>|T-2|$, that is, $\Delta>(T-2)^{2}$. Note that this implies $\Delta>0$. Since $\Delta>(T-2)^{2}$ is equivalent to $T>D+1$, the proof is complete.

Lemma 4.6. (1) If $\alpha>4$ then $(0,0)$ and

$$
q=\left(\sqrt{1-\frac{4}{\alpha}}, \sqrt{1-\frac{4}{\alpha}}\right)
$$

are fixed points of $f$. If $\alpha>2(1+\sqrt{2})$ then additionally

$$
q_{1}=\left(\frac{\alpha+\sqrt{\alpha^{2}-4 \alpha-4}}{2(\alpha+1)}, \frac{\alpha-\sqrt{\alpha^{2}-4 \alpha-4}}{2(\alpha+1)}\right)
$$

and

$$
q_{2}=\left(\frac{\alpha-\sqrt{\alpha^{2}-4 \alpha-4}}{2(\alpha+1)}, \frac{\alpha+\sqrt{\alpha^{2}-4 \alpha-4}}{2(\alpha+1)}\right)
$$

are fixed points of $f$. These are all fixed points of $f$.

(2) If $4<\alpha<2(1+\sqrt{2})$ then $q$ is a saddle; if $\alpha>2(1+\sqrt{2})$ then $q$ is attracting.

(3) Points $q_{1}$ and $q_{2}$ are saddles.

Proof. Let us find all fixed points of $f$. For this we have to solve the system of equations

$$
\begin{aligned}
& x=\frac{-2 y+\alpha x(1+y)}{2+\alpha x(1+y)} \\
& y=\frac{-2 x+\alpha y(1+x)}{2+\alpha y(1+x)} .
\end{aligned}
$$


It can be rewritten as

$$
\begin{aligned}
& 2 x+2 y=\alpha x(1+y)(1-x), \\
& 2 x+2 y=\alpha y(1+x)(1-y) .
\end{aligned}
$$

Therefore we get

$$
x(1+y)(1-x)=y(1+x)(1-y),
$$

which is equivalent to

$$
(y-x)(x y+x+y-1)=0 .
$$

This means that either $x=y$ or $x y=1-x-y$.

If $x=y$ then (4.7) becomes $4 x=\alpha x\left(1-x^{2}\right)$ which has non-negative solutions $x=0$ and $x=\sqrt{1-\frac{4}{\alpha}}$. This proves that if $\alpha>4$ then $(0,0)$ and $q$ are fixed points of $f$.

If $x y=1-x-y$ then (4.7) is equivalent to $x+y=\alpha x y$. Plugging this value of $x+y$ back to the equation $x y=1-x-y$ we get $x y=1 /(\alpha+1)$ and $x+y=\alpha /(\alpha+1)$. This means that $x$ and $y$ are the two roots of the equation

$$
x^{2}-\frac{\alpha}{\alpha+1} x+\frac{1}{\alpha+1}=0 .
$$

This equation has real roots if $\alpha \geq 2(1+\sqrt{2}$ ) (where for $\alpha=2(1+\sqrt{2}$ ) both roots are $\sqrt{1-4 / \alpha}$ ), and this gives us additional fixed points $q_{1}$ and $q_{2}$ for $\alpha>2(1+\sqrt{2})$.

The method we used gave us all solutions of our system of equations, so statement (1) is proved.

To prove statements (2) and (3), we need the partial derivatives of $f$. They are:

$$
\begin{aligned}
\frac{\partial x^{\prime}}{\partial x} & =\frac{2 \alpha(1+y)^{2}}{[2+\alpha x(1+y)]^{2}}, \\
\frac{\partial x^{\prime}}{\partial y} & =\frac{-4}{[2+\alpha x(1+y)]^{2}}, \\
\frac{\partial y^{\prime}}{\partial x} & =\frac{-4}{[2+\alpha y(1+x)]^{2}}, \\
\frac{\partial y^{\prime}}{\partial y} & =\frac{2 \alpha(1+x)^{2}}{[2+\alpha y(1+x)]^{2}} .
\end{aligned}
$$

Thus, the eigenvalues of the derivative of $f$ at $q$ are

$$
\frac{2 \alpha(1+x)^{2}-4}{[2+\alpha x(1+x)]^{2}},
$$

corresponding to the eigenvector $(1,1)^{T}$, and

$$
\frac{2 \alpha(1+x)^{2}+4}{[2+\alpha x(1+x)]^{2}},
$$

corresponding to the eigenvector $(1,-1)^{T}$, where $x=\sqrt{1-4 / \alpha}$. Note that both eigenvalues are positive. Simple computations (where we replace $x^{2}$ by $1-4 / \alpha$ whenever it appears) show that the first eigenvalue is smaller than 1 if and only if

$$
\alpha^{2}-6 \alpha+8>\left(4 \alpha-\alpha^{2}\right) x \text {. }
$$


The left-hand side is equal to $(\alpha-4)(\alpha-2)$, so for $\alpha>4$ it is positive, while the right-hand side is negative. This shows that the first eigenvalue is smaller than 1. Similarly, the second eigenvalue is smaller than 1 if and only if

$$
\alpha^{2}-6 \alpha+4>\left(4 \alpha-\alpha^{2}\right) x .
$$

If $\alpha \geq 3+\sqrt{5}$ then the left-hand side is non-negative, while the right-hand side is negative, so the second eigenvalue is smaller than 1 . If $4<\alpha<3+\sqrt{5}$ then both sides are negative, so we take their squares and change the direction of the inequality. We get after a short computation $\alpha^{2}-4 \alpha-4>0$, which is true for $\alpha>2(1+\sqrt{2})$. Thus, the second eigenvalue is smaller than 1 if $\alpha>2(1+\sqrt{2})$ and greater than 1 if $4<\alpha<2(1+\sqrt{2})$. This proves statement (2).

Consider now the fixed point $q_{1}=(x, y)$ (or $q_{2}=(x, y)$; the computations are the same). As we established in the proof of statement (1),

$$
x y+x+y=1
$$

and $x, y$ are the two roots of the equation (4.9). Therefore,

$$
x y=\frac{1}{\alpha+1}, \quad x+y=\frac{\alpha}{\alpha+1}
$$

and

$$
x^{2}=\frac{\alpha x-1}{\alpha+1}, \quad y^{2}=\frac{\alpha y-1}{\alpha+1} .
$$

In view of $(4.10)$, we have $x(1+y)=1-y$ and $y(1+x)=1-x$, so the derivative of $f$ at $q_{1}$ is

$$
\left[\begin{array}{cc}
\frac{2 \alpha(1+y)^{2}}{[2+\alpha(1-y)]^{2}} & \frac{-4}{[2+\alpha(1-y)]^{2}} \\
\frac{-4}{[2+\alpha(1-x)]^{2}} & \frac{2 \alpha(1+x)^{2}}{[2+\alpha(1-x)]^{2}}
\end{array}\right] .
$$

Its determinant is positive, so by Lemma 4.5 in order to prove statement (3) it remains to check that its trace is larger than its determinant plus 1 . This is equivalent to

$$
\left[2 \alpha(1+y)^{2}-(2+\alpha(1-y))^{2}\right]\left[2 \alpha(1+x)^{2}-(2+\alpha(1-x))^{2}\right]<16 .
$$

Using (4.12) we get

$$
2 \alpha(1+y)^{2}-(2+\alpha(1-y))^{2}=\frac{\left(\alpha^{3}+12 \alpha^{2}+8 \alpha\right) y-\left(\alpha^{3}+2 \alpha^{2}+8 \alpha+4\right)}{\alpha+1}
$$

and

$$
2 \alpha(1+x)^{2}-(2+\alpha(1-x))^{2}=\frac{\left(\alpha^{3}+12 \alpha^{2}+8 \alpha\right) x-\left(\alpha^{3}+2 \alpha^{2}+8 \alpha+4\right)}{\alpha+1} .
$$

Thus, (4.13) is equivalent to

$$
(s y-t)(s x-t)<16(\alpha+1)^{2},
$$

where

$$
s=\alpha^{3}+12 \alpha^{2}+8 \alpha \quad \text { and } \quad t=\alpha^{3}+2 \alpha^{2}+8 \alpha+4 .
$$

By (4.11), we have

$$
(s y-t)(s x-t)=s^{2} \frac{1}{\alpha+1}-s t \frac{\alpha}{\alpha+1}+t^{2},
$$


so (4.14) is equivalent to

$$
s^{2}-\alpha s t+(\alpha+1) t^{2}<16(\alpha+1)^{3} .
$$

This in turn is equivalent to

$$
8 \alpha^{6}-8 \alpha^{5}-104 \alpha^{4}-184 \alpha^{3}-128 \alpha^{2}-32 \alpha>0
$$

The left-hand side of this inequality can be factorized as

$$
8 \alpha(\alpha+1)^{3}\left(\alpha^{2}-4 \alpha-4\right)
$$

so it is positive for $\alpha>2(1+\sqrt{2})$. This completes the proof.

Remark 4.7. A quick look at the formulas for the partial derivatives of $f$ tells us that the Jacobian of $f$ is positive in the whole square for all $\alpha>2$. Moreover, by Lemmas 3.1 and 3.3 the diagonal, the region above the diagonal and the region below the diagonal are mapped by $f$ respectively to the diagonal, above the diagonal and below the diagonal.

Lemma 4.8. The map $f$ is a diffeomorphism from $[0,1]^{2}$ onto its image.

Proof. In view of Lemma 3.6, Remark 4.7 and the symmetry of $f$ with respect to the diagonal, it is enough to show that $f$ restricted to the union of the segments $I=[0,1] \times\{0\}$ and $J=\{1\} \times[0,1]$ is a homeomorphism onto its image. On $I$ we have $y^{\prime}=-x$ and on $J$ we have $y^{\prime}=1-2 /(1+\alpha y)$. In both expressions the right-hand side is a strictly monotone function of the variable, so $f$ is one-toone, and therefore a homeomorphism onto image, on both $I$ and $J$. It remains to check that $f(I)$ and $f(J)$ are disjoint except for the common endpoint. Suppose that $f(x, 0)=f(1, y)$. Then by the formulas above, $-x=1-2 /(1+\alpha y)$. Comparing $x^{\prime}$ we get $1 /(2+\alpha x)=(1+y) /(2+\alpha(1+y))$. Eliminating $x$ from this system of two equations we get

$$
\frac{1+\alpha y}{2+\alpha+2 \alpha y-\alpha^{2} y}=\frac{1+y}{2+\alpha+\alpha y} .
$$

This is equivalent to $y(\alpha-1)(\alpha y+\alpha+1)=0$. Therefore the only solution is $y=0$, which gives us $x=1$. This completes the proof.

According to Lemma 4.6, we have to distinguish between the case $4<\alpha \leq 2(1+$ $\sqrt{2}$ ), when $f$ has only one fixed point other than $(0,0)$, and the case $\alpha>2(1+\sqrt{2})$, when $f$ has three such points. Let us consider the first case (see Figure 4). For $\alpha=2(1+\sqrt{2})$ the derivative of $f$ at the fixed point $q$ has one eigenvalue equal to 1 . Therefore we need a special lemma about the nature of this point.

Lemma 4.9. Assume that $\alpha=2(1+\sqrt{2})$. Let $p \in[0,1]^{2}$ be a point that does not lie on the diagonal. Then the trajectory of $p$ does not converge to the fixed point $q$.

Proof. According to Lemma $4.6(1), q=(\sqrt{2}-1, \sqrt{2}-1)$. Let us change the variables, so that $q$ becomes the origin, one axis is along the diagonal, and the other perpendicular to it. That is,

$$
u=(x-\sqrt{2}+1)+(y-\sqrt{2}+1), \quad v=x-y .
$$

If the image of $(u, v)$ is $\left(u^{\prime}, v^{\prime}\right)$, then

$$
u^{\prime}=\frac{2\left[(2+\sqrt{2})\left(u^{4}+v^{4}\right)-(4+2 \sqrt{2}) u^{2} v^{2}+(6+10 \sqrt{2}) u\left(u^{2}-v^{2}\right)+(36+4 \sqrt{2}) u^{2}-(20+12 \sqrt{2}) v^{2}+(8+16 \sqrt{2}) u\right]}{\left[(1+\sqrt{2})\left(u^{2}-v^{2}\right)+(6+2 \sqrt{2}) u+(2+2 \sqrt{2}) v+(4+4 \sqrt{2})\right]\left[(1+\sqrt{2})\left(u^{2}-v^{2}\right)+(6+2 \sqrt{2}) u-(2+2 \sqrt{2}) v+(4+4 \sqrt{2})\right]},
$$



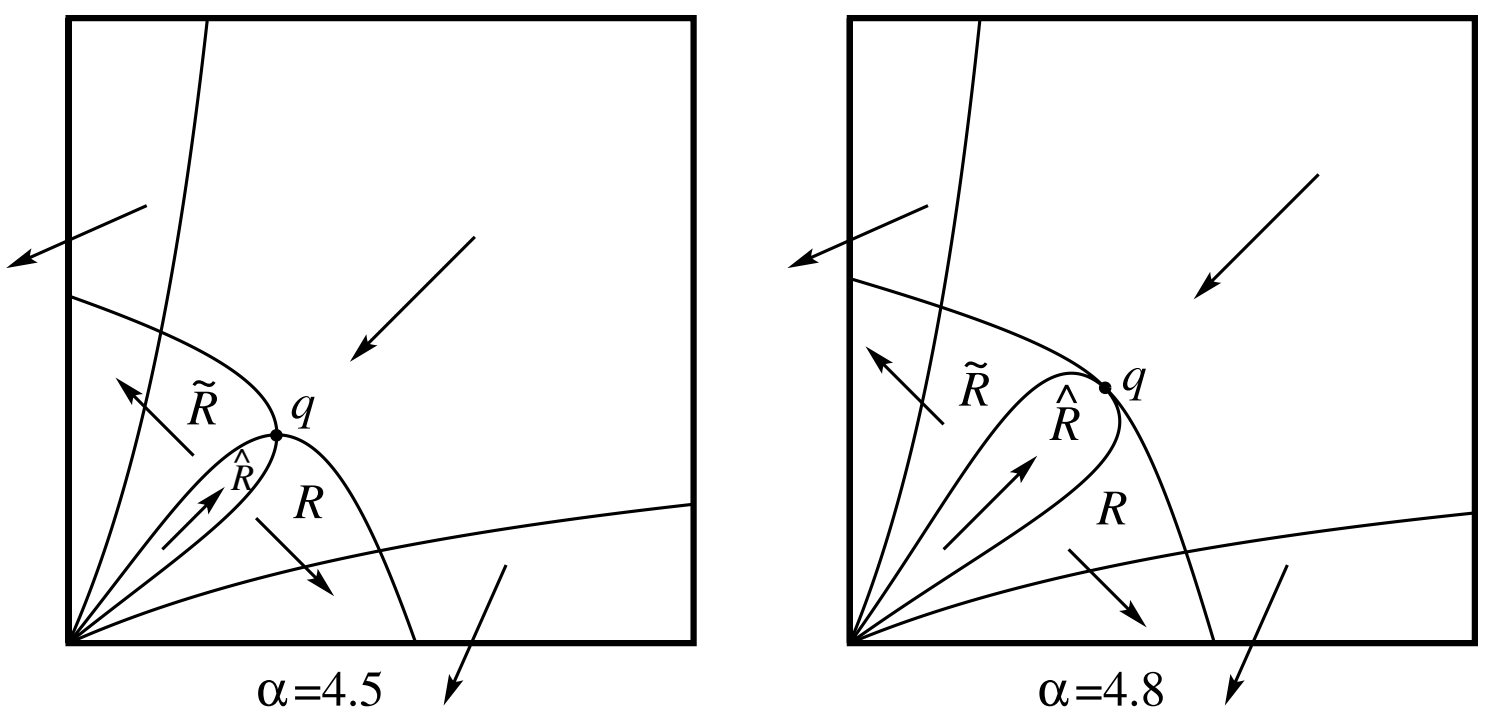

Figure 4. Map $f$ for $\alpha=4.5$ and $\alpha=4.8$

$$
v^{\prime}=\frac{4 v\left[(1+\sqrt{2})\left(u^{2}-v^{2}\right)+(8+4 \sqrt{2}) u+(12+8 \sqrt{2})\right]}{\left[(1+\sqrt{2})\left(u^{2}-v^{2}\right)+(6+2 \sqrt{2}) u+(2+2 \sqrt{2}) v+(4+4 \sqrt{2})\right]\left[(1+\sqrt{2})\left(u^{2}-v^{2}\right)+(6+2 \sqrt{2}) u-(2+2 \sqrt{2}) v+(4+4 \sqrt{2})\right]} .
$$

Taking the second order approximation to $u^{\prime}$ and $v^{\prime} / v$ we get

$$
\begin{aligned}
& u^{\prime}=(4 \sqrt{2}-5) u-\frac{19-13 \sqrt{2}}{2} u^{2}-\frac{3-\sqrt{2}}{2} v^{2}+h_{1}(u, v), \\
& v^{\prime}=v\left[1-(3 \sqrt{2}-3) u+\frac{48-31 \sqrt{2}}{4} u^{2}+\frac{4-\sqrt{2}}{4} v^{2}+h_{2}(u, v)\right]
\end{aligned}
$$

where

$$
\lim _{u, v \rightarrow 0} \frac{h_{1}(u, v)}{u^{2}+v^{2}}=\lim _{u, v \rightarrow 0} \frac{h_{2}(u, v)}{u^{2}+v^{2}}=0 .
$$

Since the numbers $19-13 \sqrt{2}, 3-\sqrt{2}, 48-31 \sqrt{2}$ and $4-\sqrt{2}$ are positive, there exists a neighborhood $U$ of $(0,0)$ such that if $(u, v) \in U$ then

$$
u^{\prime} \leq \lambda u \quad \text { and } \quad\left|v^{\prime}\right| \geq|v|(1-\mu u),
$$

where

$$
\lambda=4 \sqrt{2}-5 \quad \text { and } \quad \mu=3 \sqrt{2}-3 .
$$

Note that $0<\lambda<1$ and $\mu>0$.

Suppose that the trajectory of a point $p \in[0,1]^{2}$ that does not lie on the diagonal converges to $q$. We are working in the coordinates $u, v$, so $q=(0,0)$. By replacing $p$ by its image under a large iterate of the map we may assume that the whole trajectory is contained in $U$. Denote the $n$-th point of this trajectory by $\left(u_{n}, v_{n}\right)$. By Remark 4.7, $v_{n} \neq 0$ for all $n$. If for some $n$ we have $u_{n} \leq 0$, then by (4.15) $u_{m} \leq 0$ for all $m \geq n$, so, again by (4.15), $\left|v_{m+1}\right| \geq\left|v_{m}\right|$ for all $m \geq n$. Therefore $v_{m}$ does not converge to 0 , a contradiction. Thus, $u_{n}>0$ for all $n$. By (4.15) we get $u_{n} \leq u_{0} \lambda^{n}$ for all $n$, so, 
once more by (4.15),

$$
\left|v_{n}\right| \geq\left|v_{0}\right| \prod_{i=0}^{n-1}\left(1-\mu u_{0} \lambda^{i}\right)>\left|v_{0}\right| \prod_{i=0}^{\infty}\left(1-\mu u_{0} \lambda^{i}\right)
$$

(of course, we may assume that $\mu u_{0}<1$ ). The infinite product above is convergent to a positive number, so $\left|v_{n}\right|$ is bounded away from 0 , a contradiction. This completes the proof.

Lemma 4.10. Assume that $4<\alpha \leq 2(1+\sqrt{2})$. Then for $f$ the trajectories of all points on the diagonal except $(0,0)$ converge to the fixed point $q$. The trajectories of all points not on the diagonal leave the square $[0,1]^{2}$.

Proof. The reader is advised to consult Figure 4 when reading this proof.

By Remark 4.7, the diagonal is invariant. By Lemma 4.3, all points of the diagonal except $(0,0)$ and $q$ are mapped by $f$ in the direction of $q$. This proves that the trajectories of all points on the diagonal except $(0,0)$ converge to $q$.

Consider the set $\widehat{R}=\overline{R \cap \widetilde{R}}$ and a point $p=(x, y)$ from the part of its boundary above the diagonal. Then $p$ belongs to the boundary of $R$, so $x^{\prime}=x$. It also belongs to $\widetilde{R}$, so $y^{\prime}>y$. In other words, the vector $f(p)-p$ points vertically upwards. Since this part of the boundary is a part of the graph of the function (4.4), it is mapped by $f$ to a curve above itself. By symmetry, the part of the boundary of $\widehat{R}$ below the diagonal is mapped by $f$ to a curve to the right of itself. This proves that $f(\widehat{R}) \supset \widehat{R}$.

By Lemma 4.3, all points of $\widehat{R}$ are mapped by $f$ upwards and to the right. Therefore their trajectories either converge to $q$ or leave $\widehat{R}$. If $\alpha \leq 2(1+\sqrt{2})$, then by Lemma 4.6, $q$ is a saddle, so its stable manifold is one-dimensional. We know already this manifold, it is the diagonal (without $(0,0)$ ). Thus the trajectories of the points not on the diagonal cannot converge to $q$. Hence, they have to leave $\widehat{R}$. If $\alpha=2(1+\sqrt{2})$, we get to the same conclusion using Lemma 4.9.

If a point $p=(x, y)$ is above the diagonal and not in $\widehat{R}$, then by Lemma 4.3 $x^{\prime}<x$. Its trajectory cannot enter $\widehat{R}$, because $f(\widehat{R}) \supset \widehat{R}$ and $f$ is one-to-one (by Lemma 4.8). Therefore this trajectory either leave the square $[0,1]^{2}$ or converges to one of the fixed points $(0,0)$ and $q$. It cannot converge to $q$ by the argument from the preceding paragraph. It cannot converge to $(0,0)$, because when close to $(0,0)$, it would be in $\widetilde{R}$, where $f$ sends points upwards. Thus, it has to leave the square. By the symmetry, the trajectory of every point below the diagonal and not in $\widehat{R}$ has to leave the square. This completes the proof.

Now we move to the case $\alpha>2(1+\sqrt{2})$ (see Figure 5 ).

Lemma 4.11. Assume that $\alpha>2(1+\sqrt{2})$. Then for $f$ the stable manifold $W_{1}^{s}$ of $q_{1}$ goes from $(0,0)$ (exclusive) via $q_{1}$ to a point on the right side of the square $[0,1]^{2}$. The stable manifold $W_{2}^{s}$ of $q_{2}$ goes from $(0,0)$ (exclusive) via $q_{2}$ to a point on the upper side of the square $[0,1]^{2}$. The trajectories of all points of the region between those manifolds converge to the fixed point $q$. The trajectories of all points below $W_{1}^{s}$ and all points to the left of $W_{2}^{s}$ leave the square $[0,1]^{2}$.

Proof. The reader is advised to consult Figures 5 and 6 when reading this proof. 

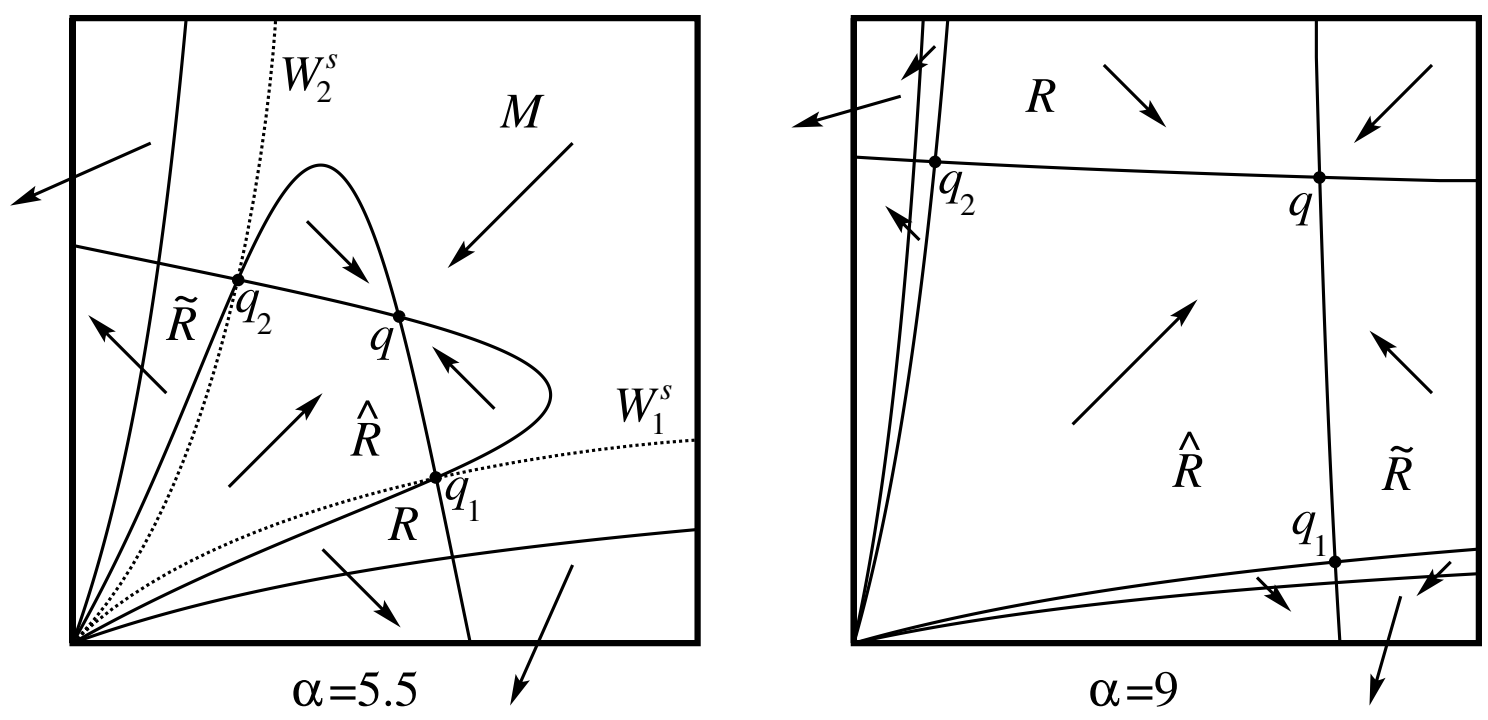

Figure 5. Map $f$ for $\alpha=5.5$ and $\alpha=9$

By a similar argument as in the proof of Lemma 4.10 (except that now we have to look at 4 pieces of the boundary of the set $\widehat{R}$, defined as in that lemma), we get $f(\widehat{R}) \supset \widehat{R}$. Moreover, arguments of the same type (looking where the vector $f(p)-p$ points on the pieces of the boundary; see Figure 6) show that each component $A$ of $R \backslash \widetilde{R}$ and of $\widetilde{R} \backslash R$ is mapped into itself or outside the square (that is, $f(A) \subseteq$ $A \cup\left(\mathbb{R}^{2} \backslash[0,1]^{2}\right)$. Here we additionally use the fact that since $f$ is an orientation preserving diffeomorphism, the circular order of the pieces of the boundaries of $R$ and $\widetilde{R}$ coming out from a fixed point remains the same after the application of $f$. The fact that for large values of $\alpha$ two of the regions have pieces of the boundary lying on the boundary of the square $[0,1]^{2}$ makes no difference, since as we noted in the proof of lemma 4.8, on the right side of the square $y^{\prime}$ is a monotone function of $y$ (and by symmetry, on the upper side of the square $x^{\prime}$ is a monotone function of $x$ ).

By Lemma 4.6, the fixed points $q_{1}$ and $q_{2}$ are saddles. They lie at the intersection points of the boundaries of $R$ and $\widetilde{R}$. Taking into account the directions in which $f$ maps the points (see Figure 5), we see that $W_{1}^{s}$ in a small neighborhood of $q_{1}$ has to lie in $\widehat{R}$ and $[0,1]^{2} \backslash(R \cup \widetilde{R})$. To see what happens to it globally, we have to take the preimages of this short piece of $W_{1}^{s}$. The set $\widehat{R}$ is invariant under $f^{-1}$, so one component of $W_{1}^{s} \backslash\left\{q_{1}\right\}$ lies in $\widehat{R}$. All points of $\widehat{R}$ are mapped by $f^{-1}$ down and to the left, so all trajectories converge to $(0,0)$. Therefore this component of $W_{1}^{s} \backslash\left\{q_{1}\right\}$ ends up at $(0,0)$ (but of course $(0,0)$ does not belong to it). The other component start in the region where all points are mapped by $f^{-1}$ up and to the right. It cannot enter a component of $\widetilde{R} \backslash R$, since that component is invariant for $f$. Therefore it ends up at a point of the upper or right side of the square. However, $W_{1}^{s}$ cannot cross the diagonal, so it has to end up at a point of the right side of the square. By symmetry, $W_{2}^{s}$ stretches from $(0,0)$ via $q_{2}$ to a point on the upper side of the square. 


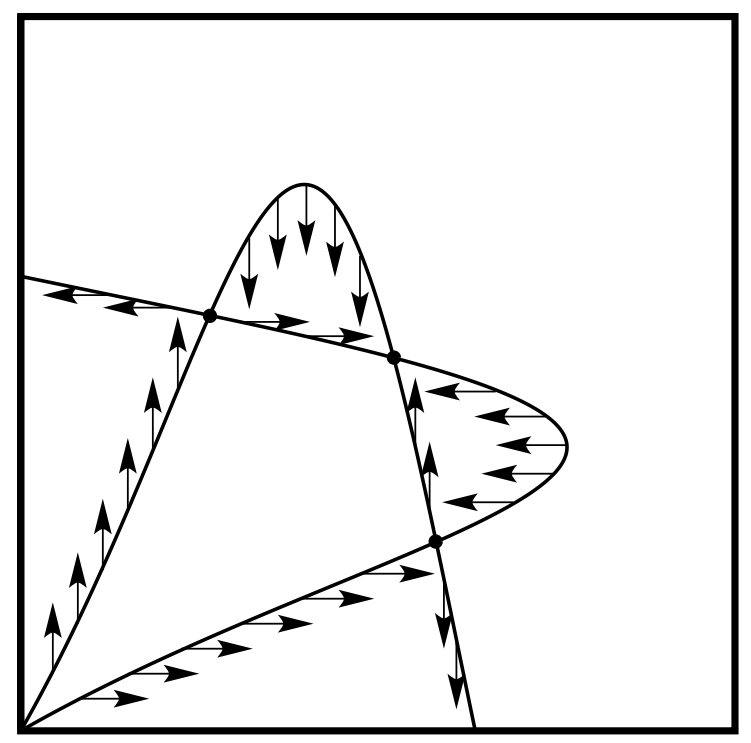

Figure 6. The directions at which the points on the boundaries of $R$ and $\widetilde{R}$ are mapped by $f$

Let $M$ be the region of $[0,1]^{2}$ between $W_{1}^{s}$ and $W_{2}^{s}$. The set $f(\widehat{R} \cap M)$ is bounded by a piece of $W_{1}^{s}$, a piece of $W_{2}^{s}$, a curve contained in the component of $R \backslash \widetilde{R}$ that lies above $\widehat{R}$ and a curve contained in the component of $R \backslash \widetilde{R}$ that lies to the right of $\widehat{R}$. The trajectories of points in those components converge to $q$ by the already standard argument about directions in which the points are mapped by $f$. The same argument shows that the trajectories contained entirely in $\widehat{R} \cap M$ do the same (they cannot converge to $q_{1}$ or $q_{2}$ because they do not lie on their stable manifolds). The same argument applies to the remaining part of $M$, that is, to $M \backslash(R \cup \widetilde{R})$. Here we use additionally the observation that such a trajectory cannot leave $M$ because it cannot cross $W_{1}^{s}$ or $W_{2}^{s}$ (remember that $f$ is an orientation preserving homeomorphism).

The trajectories of all points below $W_{1}^{s}$ and all points to the left of $W_{2}^{s}$ leave the square $[0,1]^{2}$ by the already completely standard argument and the observation that such trajectories cannot converge to $(0,0)$ (looking again at the directions). This completes the proof.

\section{Proof of the Main Theorem}

Now we are ready to prove Theorem 2.1.

Proof of (1). By Lemma 3.2 (2), $n$ has three fixed points: $(0,0),(1,1)$ and $(1 / 2,1 / 2)$. By equation (3.2), the derivative of $n$ at $(0,0)$ and $(1,1)$ is the identity, so those points preserve orientation. By Lemma 3.2 (3), they are topologically attracting.

Proof of (2). When $0<\alpha \leq 1 / 2$, then $n$ is a homeomorphism onto its image by Lemma 3.7. When $1 / 2<\alpha<2$, by Lemma 3.3 the anti-diagonal is invariant for $n$, but by Lemma $3.5 n$ restricted to it is not a homeomorphism. When $\alpha \geq 2$, by Lemma $3.5 n$ restricted to the anti-diagonal reverses orientation. Since by Lemma 3.3 
$n$ preserves the regions above and below the anti-diagonal, it reverses orientation (in two dimensions) at the points of the anti-diagonal. However, by Theorem 2.1 (1) the fixed points $(0,0)$ and $(1,1)$ are orientation preserving. Thus, $n$ cannot be a homeomorphism onto its image.

Proof of (3). Assume that $0<\alpha<2$. By Lemma 3.4 (3), (1/2,1/2) is an orientation preserving fixed saddle point. By Lemmas 3.3 and 3.5 (1), both the anti-diagonal and diagonal are invariant, so by Lemma 3.4 (2) the stable and unstable manifolds of $(1 / 2,1 / 2)$ are contained respectively in the anti-diagonal and diagonal. Since there are no other fixed points on the anti-diagonal, the stable manifold is equal to it. Since the only other fixed points on the diagonal are its endpoints, the unstable manifold is equal to the diagonal without endpoints.

Proof of (4). Assume that $2<\alpha<4$. By Lemma $3.4(3),(1 / 2,1 / 2)$ is an orientation reversing fixed saddle point. By Lemmas 3.3 and 3.5 (1), both the antidiagonal and diagonal are invariant, so by Lemma $3.4(2)$ the stable and unstable manifolds of $(1 / 2,1 / 2)$ are contained respectively in the anti-diagonal and diagonal. By Lemma 4.1, the stable manifold is equal to the anti-diagonal. Since the only other fixed points on the diagonal are its endpoints, the unstable manifold is equal to the diagonal without endpoints.

Proof of (5). This is Lemma 4.4.

Proof of (6). Assume that $4<\alpha<2(1+\sqrt{2})$. By Lemma $3.4(3),(1 / 2,1 / 2)$ is an orientation reversing repelling fixed point. By Lemma 4.6 (1), there is a period 2 orbit on the anti-diagonal (corresponding to the fixed point $q$ of $f$ ). By Lemma 4.6 (2), it is a saddle. One point of this orbit lies below $(1 / 2,1 / 2)$. By Lemma 4.10 , its stable manifold is the part of the anti-diagonal with $x<1 / 2$. By symmetry, the other point lies above $(1 / 2,1 / 2)$ and its stable manifold is the part of the anti-diagonal with $x>1 / 2$. By Lemmas 4.10, 4.2,3.2 (3) and 3.3, all points below the anti-diagonal are attracted to $(0,0)$ and all points above the anti-diagonal are attracted to $(1,1)$.

Note that if $\alpha=2(1+\sqrt{2})$, we get the same results, except that the period 2 orbit on the anti-diagonal is not a saddle.

Proof of ( 7$)$. Assume that $\alpha>2(1+\sqrt{2})$. By Lemma $3.4(3),(1 / 2,1 / 2)$ is an orientation reversing repelling fixed point. By Lemma $4.6(1)$, there is a period 2 orbit $P$ on the anti-diagonal (corresponding to the fixed point $q$ of $f$ ) and there are two period 2 orbits that follow $P$ (corresponding to the fixed points $q_{1}$ and $q_{2}$ of $f$ ). By Lemma 4.6 (2) and (3), $P$ is attracting, and the other two period 2 orbits are saddles. By the symmetries, one saddle orbit lies below the anti-diagonal and one above. By Lemmas 4.11, 4.2 and 3.2 (3), every other point is attracted to one of the periodic orbits mentioned (including $(0,0)$ and $(1,1)$ ).

\section{REFERENCES}

[1] D. Barash, The Survival Game, Owl Books, New York, 2003.

[2] R. A. Becker and S. K. Chakrabarti, "Satisficing Behavior, Brouwer's Fixed Point Theorem and the Nash Equilibrium," Economic Theory 26 (2005), 63-83. 
24 R. A. BECKER, S. K. CHAKRABARTI, W. GELlER, B. KITCHENS, AND M. MISIUREWICZ

[3] R. A. Becker, S. K. Chakrabarti, W. Geller, B. Kitchens, and M. Misiurewicz, "Dynamics of the Nash Map in the Game of Matching Pennies," Journal of Difference Equations and Applications 13 (2007), 223-235.

[4] W. R. Derrick, "A Condition Under Which a Map is a Homeomorphism," American Mathematical Monthly 80 (1973), 554-555.

[5] J. F. Nash, Non-Cooperative Games, Ph.D. dissertation, Princeton University, 1950.

[6] J. F. Nash, "Equilibrium Points in n-Person Games," Proceedings of the National Academy of Sciences 36 (1950), 48-49.

[7] J. F. Nash, "Non-Cooperative Games," Annals of Mathematics 54 (1951), 286-295.

[8] K. Ritzberger and J. Weibull, "Evolutionary Selection in Normal-Form Games," Econometrica 63 (1995), 1371-1399.

[9] B. Skyrms, The Stag Hunt and the Evolution of Social Structure, Cambridge University Press, Cambridge, UK, 2004.

Department of Economics, Indiana University, Bloomington, IN 47405

E-mail address: becker@indiana.edu

Department of Economics, Indiana University - Purdue University Indianapolis, 425 University Blvd., Indianapolis, IN 46202

E-mail address: imxl100@iupui.edu

Department of Mathematical Sciences, Indiana University - Purdue University Indianapolis, 402 N. BlackFord Street, Indianapolis, IN 46202

E-mail address: wgeller@math.iupui.edu

Department of Mathematical Sciences, Indiana University - Purdue University Indianapolis, 402 N. BlackFord Street, Indianapolis, IN 46202

E-mail address: bkitchens@math.iupui.edu

Department of Mathematical Sciences, Indiana University - Purdue University Indianapolis, 402 N. Blackford Street, Indianapolis, IN 46202

E-mail address: mmisiure@math.iupui.edu 